\title{
Can Previous Pro-Environmental Behaviours Influence Subsequent Environmental Behaviours? The Licensing Effect of Pro-Environmental Behaviours
}

\author{
Liuna Geng, ${ }^{1}$ Xiao Cheng, ${ }^{1}$ Zhuxuan Tang, ${ }^{1}$ Kexin Zhou, ${ }^{2}$ and Lijuan Ye ${ }^{1}$ \\ ${ }^{1}$ Department of Psychology, Nanjing University, Nanjing, China \\ ${ }^{2}$ Nanjing Institute of Environmental Sciences, Ministry of Environmental Protection, Nanjing, China
}

\begin{abstract}
M ethods for promoting individuals' pro-environmental behaviours are becoming very important. Besides traditional factors relevant to pro-environmental actions, few studies have examined the licensing effect within this domain. Across three studies we have explored whether the licensing effect really exists in pro-environmental behaviours, how this effect actually works, and how to inhibit or even avoid this effect. Results have revealed that previous pro-environmental behaviours lead to a decrease in subsequent pro-environmental behaviours, indicating a licensing effect. Also, the licensing effect in pro-environmental behaviours can be explained by having individuals focus on goal progress and reduced by focusing on goal commitment. Furthermore, attributional recall could be an intervention for mitigating the licensing effect.
\end{abstract}

Keywords: pro-environmental behaviour, licensing effect, goal commitment, goal progress

In today's global community, human-driven environmental problems have wide-reaching implications for many domains, such as climate change, air quality, water and noise pollution, depletion of the ozone layer, energy consumption, and loss of biodiversity (Steg \& Velk, 2009; Tiefenbeck et al., 2013; Hautier et al., 2015). Within these scenarios, individuals are facing more extreme environmental events that could be detrimental to our overall life quality (Dominicis, Fornara, Cancellieri, Twigger-Ross, \& Bonaiuto, 2015). Hence, it is important to find interventions for improving current environments that can be applied throughout the whole world. Some researchers argue that many of our environmental issues could be addressed by changing people's behaviours (Steg \& Velk, 2009). Thus, many scholars have advocated for implementing interventions that could boost pro-environmental behaviours and further improve the environment (Truelove et al., 2014). A wealth of researches have attempted to develop practical methods for facilitating behaviour change (Steg \& Velk, 2009) or to find out key factors necessary for pro-environmental lifestyle advocacy (Steg \& Velk, 2009; Canuel, Abdous, Bélanger \& Gosselin, 2014).
As is well known, pro-environmental behaviour does not take place in a vacuum, but often occurs in everyday life. This means that many of our regular behaviours, such as water and electricity consumption, are related to environmental protection. Therefore, it is not enough to simply focus on promoting just one possible proenvironmental behaviour, we also need to pay attention to the continuity of pro-environmental behaviours. In this regard, few studies have found that past behaviour can influence future behaviour. Specifically, there are questions as to whether past pro-environmental behaviours will promote or inhibit future pro-environmental actions (Van der Werff, Steg, \& Keizer, 2014). According to cognitive dissonance theory (Festinger, 1957), individuals tend to maintain consistency with behaviour they have performed before; otherwise, they will experience a sense of discomfort or uneasiness. Some previous studies about pro-environmental behaviours have shown this outcome. For example, a study revealed that individuals who reused paper were more likely to avoid excessive packaging (Thøgersen, 1999); similar results were obtained with water and energy conservation, as well as recycling plastic

Address for correspondence: Dr Liuna Geng, Department of Psychology, Nanjing University, Nanjing, China. Email: gengliuna@nju.edu.cn 
bags (Berger, 1997). In contrast, recent studies have revealed a phenomenon of inconsistent behaviours wherein a past pro-environmental behaviour actually inhibits a future pro-environmental behaviour, which is referred to as the licensing effect. In one study, Clot, Grolleau, and Ibanez (2014) found that when participants performed pro-environmental behaviours, their willingness to donate to an environmental charity would reduce. Tiefenbeck, Staake, Roth, and Sachs (2013) observed that people who reduced water consumption actually increased their electricity usage later. There was additional evidence of individuals being selectively pro-environmental, such as behaving pro-environmentally at home but less so while travelling (Barr, Shaw, Coles, \& Prillwitz, 2010). Additionally, consumers might increase their consumption levels if they are informed beforehand that the products they intend to use are recyclable (Bolton, Cohen, \& Bloom, 2006). Therefore, important questions remain as to whether proenvironmental behaviours are subject to the licensing effect rather than remaining consistent, and if so, why previous pro-environmental behaviours lead to subsequent reductions.

Drawing on previous research assessing the licensing effect, a few researchers have speculated about why past pro-environmental behaviours weaken subsequent green behaviours (Mullen \& Monin, 2016). Van der Werff et al. (2014) and Meijers (2014) suggested that the level of an individual's environmental self-identity (i.e., the extent to which an individual identified him or herself as an environmentally friendly person) might matter. According to their assumption, past pro-environmental behaviours could signal individuals' identity. If the previous actions signal participants' environmental self-identity weakly, their subsequent behaviours would show a licensing effect. That is, participants with a weaker environmental self-identity would show more significant licensing effects in their pro-environmental behaviours. While this could provide an explanation for the licensing effect, it cannot be applied to all possible situations, due to different behaviours having different functions in terms of signalling self-identity. For instance, reducing water consumption and eating green food are both pro-environmental behaviours, but they cannot signal the same level of environmental self-identity; hence, the subsequent behaviours would be different or even opposite. Thus, for the present study, we argue that the goal-regulation theoretical framework proposed by Fishbach and Dhar (2005) accounts for the psychological mechanism involved in people's subsequent environmental behaviours. Individuals take action according to the goals they hold; hence, Fishbach abd Darh argue that whether a self-licensing effect emerges could be explained by an individual's goal focus. In terms of goal regulation, the same movement towards one goal could be considered as either goal progress or goal commitment by different individuals (Mullen \& Monin, 2016). Regarding goal progress, individuals consider their previous goalrelated behaviour as evidence of having made progress to the goal, which could produce a sense of achievement and goal fulfilment. Consequently, they shift their cognitive resources away from this initial goal and focus on achieving other goals (Dhar \& Simonson, 1999; Monin \& Miller, 2001; Fishbach \& Dhar, 2005). However, in terms of goal commitment, previous goal-related behaviour can be regarded as evidence of commitment to the goal, which could still maintain cognitive resources focused on the goal, strengthening one's firm determination to achieve the goal. Thus, individuals are motivated to engage in reducing inconsistent behaviours and inhibiting other goals (Shah, Kruglanski, \& Friedman, 2003; Fishbach, Dhar, \& Zhang, 2006). Applying this theory to the environmental behaviour domain, when an individual focuses on goal progress, subsequent pro-environmental behaviours would be reduced after performing a pro-environmental action, indicating a licensing effect. However, when focusing on goal commitment, the licensing effect would be decreased or even be replaced by consistently environmentally friendly behaviours.

The current study focused on methods for inhibiting or even eliminating the occurrence of a licensing effect in pro-environmental behaviours. Fishbach et al. (2006) suggested that people who focused on goal progress may transition to an alternative goal when they thought adequate progress had been made toward the initial goal (environmental protection), as described above. According to goal-regulation theory, we could make people shift their focus to goal commitment and concentrate their cognitive resources on the initial goal (environmental protection), ultimately reducing licensing effects in pro-environmental behaviours (Fishbach, Zhang, \& Koo, 2009). Consequently, we designed a priming task that involved attributional recall to change participants' goal focus. Mukhopadhyay, Sengupta, and Ramanathan (2008) observed that the activation of a goal can be enhanced at the point of recalling attributions of previous goal-related actions. By reminding individuals of the reasons for why they previously performed the behaviours, recalling attributions could highlight their commitment towards the goal and concentrate more cognitive resources on it; the reinforcement of goal cognition should help inhibit other competing goals (Shah \& Kruglanski, 2003). Therefore, recalling attributions could reduce the intention of performing inconsistent behaviours related to that initial goal, that is, inhibiting the licensing effect.

In sum, our ultimate goal in the present study was to examine plausible interventions for reducing or even avoiding a licensing effect in ongoing pro-environmental behaviours. The present study consisted of three substudies. Study 1 employed a virtual shopping task and discusses whether the licensing effect actually exists in environmental behaviours. Study 2 aimed to use goal-regulation theory to explain the psychological mechanism of the licensing effect in the environmental protection field and explore a way to reduce it. Finally, Study 3 was implemented to outline an intervention for alleviating 
the licensing effect, so as to promote consistent proenvironmental behaviours.

\section{Study 1: Licensing Effect Related to Pro-Environmental Behaviours}

In Study 1, we tested whether participants who previously performed a pro-environmental behaviour would reduce their subsequent environmental protection behaviours. Two experiments were implemented for Study 1: 1a measured subsequent behaviour using a behavioural experiment of water consumption; while $1 \mathrm{~b}$ used a questionnaire assessment about daily pro-environmental behaviours. We hypothesised that the participants who previously performed pro-environmental behaviours would show reduced green actions compared to participants who had not performed pro-environmental behaviours, indicating a licensing effect.

\section{Method}

Participants and procedure. All respondents were students at Nanjing University who were randomly recruited from flyers posted around campus. In total, 80 participants participated in Study 1, of whom 26 were male. Ages ranged from 17 to $28(M=22.18, S D=2.50)$. The Institutional Review Board at Nanjing University approved all experimental protocols. Forty students took part in the survey for Experiment 1a. Participants were assigned to complete the shopping list (20 were given the list with nine green products and three conventional products; 20 were given the opposite list), which was designed to manipulate the green behaviours performed. Next, participants cleaned their towel and completed the evaluation assessment. After the experiment, participants were given the towel as compensation for participating. The task of cleaning the towel was actually done to measure water consumption, and we hypothesised that purchasing more green products was expected to lead to greater water consumption. Another 40 participants took part in Experiment $1 \mathrm{~b}$. These participants also first completed the shopping list and then completed the environmental behaviour questionnaire. Experiment 1a was designed to measure participants' real behaviours about environmental protection, and the other experiment was designed to test their intended pro-environmental behaviours.

\section{Materials}

Purchasing task. According to Mazar and Zhong (2010), the purchasing task is intended to simulate the process of shopping in a supermarket. Participants had to complete a shopping list for preferred products. There were two kinds of shopping lists, and participants were randomly assigned to either one. One list included nine green products and three conventional products. The other list consisted of three green products and nine conventional products. The two shopping lists were equivalent in product quantity, product category, product price, and product shape. For green products, descriptors focused on proenvironmental performance; for conventional products, descriptors focused more on information regarding applications. The design of the two shopping lists influenced the number of green products participants purchased. All participants were given an imaginary budget of RMB 70 (total price for the entire list was RMB 132) and told to purchase products based on need.

Water consumption task. Tiefenbeck et al. (2013) utilised water consumption as a valid pro-environmental behaviour. Moreover, we adopted components from Catlin and Wang's (2012) study on paper usage to assess water consumption during a towel-washing task. Participants were told that they were to help evaluate the quality of towels that appeared on the shopping lists. The goal was to examine the connection between the previous purchasing products task and participants' subsequent behaviour. Before the study, a new towel, a washbasin, and a 5-point scale that included assessments about the comfort, mellowness, and absorbency of the towel were provided on a desk, with an empty bucket under the desk. Upon arrival, participants received water from the tap by themselves at first, then washed the towel and wrung it out so that it was as dry as possible. Afterwards, participants poured water into the bucket and filled in the scale for evaluating its quality. The weight of the water in the bucket was the index of water consumption.

Assessing individual differences in environmental behaviour. We examined individuals' intentions to engage in pro-environmental behaviours. Specifically, the present study employed the Questionnaire of College Students' Environmental Behaviour (QCSEB; Shen, 2008). The questionnaire contains 23 items that relate to different daily activities, of which 14 include activities related to pro-environmental behaviours. These behaviours include using environmentally friendly products, recycling, green product use, and so on. Participants are asked to evaluate the probability of engaging in these behaviours during the next 3 months on a 6-point Likert scale ranging from 1 (extremely improbable) to 6 (highly probable). Higher total scores indicate a greater likelihood of engaging in pro-environmental behaviours. The scale has a high level of internal consistency $(\alpha=.76)$ and adequate construct validity, as revealed by relatively low correlations among different items and a relatively high correlation between each item and the final score.

\section{Results}

Across both experiments, significant differences in purchasing decisions emerged. Participants who received the conventional shopping list bought fewer proenvironmental products (Experiment 1a: $M=0.95$, $S D=0.60$; Experiment $1 \mathrm{~b}: M=0.95, S D=1.00)$ than participants given the 'green' shopping list (Experiment 1a: $M=3.50, S D=1.32$; Experiment $1 \mathrm{~b}: M=3.20, S D=$ $1.28), t(38)=-7.87, p<.01$, and $t(38)=-6.19, p<.01$, 
respectively. Therefore, the manipulations were effective in distinguishing variable environmental behaviours.

Results revealed a significant group difference in the water consumption task in Experiment 1a, $t(38)=-2.13$, $p=.04$, and the environmental behaviour questionnaire from Experiment $1 b, t(38)=2.16, p=.04$. Specifically, participants in the green group consumed more water $(M=2.13, S D=0.60)$ than the conventional group $(M=$ $1.78, S D=0.36)$. Furthermore, the green group had lower scores $(M=51.8, S D=5.54)$ on the QCSEB than the conventional group $(M=57.0, S D=9.25)$. Thus, in terms of actual and intended behaviours, participants who bought more green products reduced their pro-environmental behaviour tendencies.

The present findings supported our initial predictions. Specifically, a licensing effect emerged within our environmental behaviour manipulation. Individuals who engaged in green purchasing behaviours showed diminished subsequent environmentally friendly behaviours.

\section{Study 2: Goal Regulation and the Licensing Effect}

In Study 2, we attempted to use goal regulation theory to explore the mental mechanism for the licensing effect emerging in the environmental behaviours, and further found ways to reduce the licensing effect. According to goal regulation theory, individuals regard their previous behaviours as representing either progress or commitment towards their goals. Furthermore, differences in goal focus could lead to different outcomes. We hypothesised that participants who focus on goal progress would consider that they have achieved much progress in environment protection based on their past green action, and thus show a licensing effect for pro-environmental behaviours. However, participants who focus on goal commitment would show a different performance pattern. Their past pro-environmental behaviour could strengthen cognitions concerning environmental goals, alleviating the licensing effect and leading them to undertake behaviours more consistent with their previous pro-environmental actions. As well as demonstrating the licensing effect in the environmental domain, we also wanted to find differences in subsequent behaviours resulting from differences in goal focus.

\section{Participants and Procedure}

All respondents were students at Nanjing University randomly recruited from flyers posted around campus. In total, 167 participants participated in Study 2, of whom 94 were male. Ages ranged from 18 to $28(M=21.04$, $S D=1.87)$. The Institutional Review Board at Nanjing University approved all experimental protocols.

Study 2 included a 2 (green products vs. conventional products) $\times 2$ (goal progress vs. goal commitment) design . As with Study 1, participants first completed the shopping list task; then they were primed with a goal progress or goal commitment manipulation. Finally, participants completed the QCSEB.

\section{Materials}

Goal progress and goal commitment. Following procedures used by Susewind and Hoelzl (2014), we activated participants' focus on either goal progress or goal commitment. In terms of goal progress, participants recalled their previous shopping behaviours and were then asked to evaluate the level of goal accessibility (or goal advancement) on a 5-point Likert scale ranging from 1 (no progress) to 5 (much progress). To help minimise suspicion regarding the experimental aims, participants had to evaluate their progress toward seven goals: one personal goal, three practical goals, and three pro-environmental goals. The personal and practical goals were self-identified by participants. The pro-environmental goals were provided. For analyses, scores were averaged across the three proenvironmental goals.

In terms of goal commitment, participants also recalled their previous shopping behaviours, but they rated their level of commitment toward seven goals (again, one personal goal, three practical goals, and three proenvironmental goals). The task required participants to evaluate the importance of these goals and their impact on participants' purchasing decisions. As with the goal progress metric, an average score of the three proenvironmental goals was used as the goal commitment index.

\section{Results}

As Study 1 showed, the purchasing task manipulation was effective $\left(t_{\text {goal progress }}=3.61, p<.01 ; t_{\text {goal commitment }}=6.61\right.$, $p<.01)$. Across both groups, significant differences between goal regulation task scores emerged. In order to distinguish the shopping list groups conveniently, we called participants who received the conventional shopping list the 'non-licence group', and others who received the green shopping list the 'licensing group'. In the priming goal progress condition, participants in the non-licence group scored lower $(M=2.82, S D=0.82)$ than participants in the licensing group $(M=3.65, S D=0.65), t(83)=5.16$, $p<.01$. Similarly, under the condition of priming goal commitment, the non-licence group scored higher $(M=$ $3.83, S D=0.81)$ than the licensing group $(M=3.73$, $S D=0.53), t(80)=-.713, p<.01$ Therefore, the manipulations were effective in shifting participants' goal focus onto either progress or commitment towards the goal.

The data were screened for outliers according to the principle of 3 standard deviations above or below the mean scores, and we found one outlier in the questionnaires. Hence, the last total sample was 112 subjects. Results revealed that product type (green vs. conventional) had a significant effect on the intention of pro-environmental behaviour, $F_{(1,163)}=4.22, p<.05$. The licensing group had lower intentions $(M=55.85, S D=8.85)$ than the 
non-licence group $(M=58.56, S D=8.01)$, similar to Study 1. Additionally, goal type (goal progress vs. goal commitment) significantly affected pro-environmental behaviour intentions, $F_{(1,163)}=32.96, p<.01$. Participants who were focused on goal commitment were willing to perform more subsequent pro-environmental behaviours $(M=60.62, S D=7.25)$ than those who were concerned with goal progress $(M=53.70, S D=8.47)$. However, there was a significant interaction, $F_{(1,163)}=$ $18.27, p<.01$. In the goal progress group, participants who received the green shopping list had lower QCSEB scores $(M=50.44, S D=1.10)$ than those receiving the conventional shopping list $(M=57.70, S D=1.17)$, $F_{(1,163)}=20.37, p<.01$. Hence, goal progress participants receiving green shopping lists were more likely to show a licensing effect. This pattern was not revealed in the goal commitment group, $F_{(1,163)}=2.42, p=.122$.

The aforementioned interaction supports our hypothesis: different goal foci can lead to divergent outcomes in terms of environmentally friendly behaviours. When an individual is focused on goal progress, subsequent proenvironmental behaviours are diminished if an initial environmentally friendly action has been produced. Furthermore, goal commitment can reduce the tendency to perform behaviours harmful to the environment, indicating that the licensing effect was alleviated.

\section{Study 3: Attributions Recall and the Licensing Effect}

Based on the results of Study 2, we wanted to find a way to reduce or even avoid licensing effects due to a focus on goal progress, that is, to reduce subsequent inconsistent behaviours and even transform them into consistent behaviours. With the task proposed before, we hypothesised that attributions recall could remind individuals of their initial goal by recalling the reasons for previous goalrelated actions, and they would concentrate their cognitive resources on the initial goal, making their subsequent behaviours more consistent with previous actions.

\section{Participants and Procedure}

All respondents were students at Nanjing University randomly recruited from flyers posted around campus. In total, 162 participants participated in Study 3, of whom 76 were male. Ages ranged from 18 to $36(M=21.47$, $S D=2.77)$. The Institutional Review Board at Nanjing University approved all experimental protocols.

All participants were informed that they would be completing four unrelated tasks via a questionnaire. Participants in Experiment 3A first completed the shopping task (same as Study 1; 40 participants received the green products list, and another 40 received the conventional list). Next, participants performed the goal progress priming task. Finally, participants were instructed to recall the reason related to their previous green product purchases and then complete the QCSEB. Though Experiment 3A had supported our hypothesis that attributional recall can reduce licensing effect in pro-environmental behaviours, to further identify its influence, we administered Experiment 3B. Experiment 3B also had four parts. The first two phases were the same as in Experiment 3A; however, after the goal progress priming task, participants were divided into two groups (41 participants in both groups). One group was given the recalling goal commitments task, and the other group read a scientific paper unrelated to environmental issues.

\section{Materials}

Attributional recall. We employed a task used by Mukhopadhyay et al. (2008) that had participants recall previous experiences with purchasing pro-environmental products. Next, participants listed three reasons for why they purchased those products. If there was no previous experience, reasons for never purchasing these products were reported.

\section{Results}

As Study 2 showed, the purchasing task manipulation was effective $\left(t_{3 \mathrm{~A}}=-8.81, p_{3 \mathrm{~A}}<.01 ; t_{3 \mathrm{~B}}=-4.23, p_{3 \mathrm{~B}}<.01\right)$. Across both experiments, significant differences between the goal progress task scores emerged. Participants in the non-licence group scored lower $\left(M_{3 \mathrm{~A}}=2.93, S D_{3 \mathrm{~A}}=\right.$ $\left.0.95 ; M_{3 \mathrm{~B}}=2.87, S D_{3 \mathrm{~B}}=0.83\right)$ than participants in the licensing group $\left(M_{3 \mathrm{~A}}=3.36, S D_{3 \mathrm{~A}}=0.82 ; M_{3 \mathrm{~B}}=3.78\right.$, $\left.S D_{3 \mathrm{~B}}=0.93\right), t_{3 \mathrm{~A}}=4.94, p_{3 \mathrm{~A}}<.01, t_{3 \mathrm{~B}}=5.23, p_{3 \mathrm{~B}}<$ .01. Therefore, the manipulations were effective in shifting participants' goal focus onto progress towards the goal.

In terms of the results from Experiment 3A, no significant differences emerged between the licensing group $(M=58.72, S D=5.10)$ and the non-licence group $(M=57.05, S D=9.78)$ on QCSEB scores, $t(78)=-.960$, $p=.340$. According to the result from Study 2, participants who focused on goal progress showed licensing effects in their subsequent behaviour; that is, those who purchased more green products scored lower than those who received the conventional shopping list. This suggests that the attribution recalling task can alleviate the influence of the licensing effect. To further confirm this result, we compared the results of the goal progress group in Study 2 to those in Study 3A. Significant differences were found between the results from Study $2(M=53.70, S D=8.47)$ and Study $3 \mathrm{~A}(M=57.88, S D=7.84), t(164)=4.03, p<$ .01 . The only difference between the goal progress group of Study 2 and Study $3 \mathrm{~A}$ is that the later added an attributions recall task. This means the attribution recalling task was effective in eliminating the licensing effect.

To eliminate the possibility that the manipulation of the attribution recall task produced the different results, we compared QCSEB scores between participants who recalled attributions related to purchasing green products and participants who read an unrelated scientific paper (Experiment 3B). As expected, participants who engaged 
in the recall of goal commitments task provided higher scores $(M=58.29, S D=8.93)$ than those who read the scientific paper $(M=53.98, S D=5.64), t(80)=2.616$, $p=.011$.

As predicted, the licensing effect on participants' subsequent environmental behaviours was reduced when they recalled attributions of their previous pro-environmental purchases. Thus, recalling attributions may alleviate the licensing effect related to pro-environmental behaviours and even promote consistency in green actions.

\section{Discussion}

The present study investigated the extent to which previous environmental behaviours influenced subsequent environmentally friendly actions. Of particular importance was examining group differences in subsequent behaviour between individuals exposed to green consumer products versus those predominantly exposed to more conventional products. Additionally, we examined whether significant differences would emerge based on a particular goal focus. Namely, that different goal foci could result in different subsequent behaviours.

\section{A Licensing Effect Can Emerge With Pro-Environmental Behaviours}

According to various theoretical perspectives, behaviour can result from multiple motivations (Steg \& Velk, 2009), including self-value, beliefs (value-belief-norm theory; Stern, 2000), attitudes, perceived behavioural control (theory of planned behaviour; Ajzen, 1991), and others. Hence, one's previous behaviour may predict future behaviour in certain contexts. In the current study, for both kinds of experimental tasks (water consumption experiment and survey tasks), participants who purchased green products at the start of the study showed reduced tendencies to engage in subsequent environmentally friendly behaviours. Hence, the licensing effect emerges in a proenvironmental behaviour scenario, which is consistent with previous research. For instance, Monin and Miller (2001) revealed a licensing effect in the political domain. They observed that participants who self-identified as non-racially prejudiced actually engaged in subsequent prejudicial behaviour. In another study, a reduction in prosocial behaviour was revealed after individuals were given the chance to recall past moral behaviours (Jordan, Mullen, \& Murnighan, 2009). Moreover, a few studies even observed a licensing effect in the context of environmental behaviours. For example, in a water conservation study, Tiefenbeck et al. (2013) showed that participants consumed more electrical energy after demonstrating a reduction in water consumption. Finally, individuals who spent money to plant trees for pro-environmental motives also consumed more electrical power afterwards (Gans \& Groves, 2009). The above studies revealed licensing effects over a relatively long period (i.e., electrical energy consumption during a month). While long-term pro- environmental behaviours are important, green actions occur frequently in daily life, as mentioned earlier, and the present study is meaningful because it reveals that a licensing effect can manifest over a very short time period.

The licensing effect in a pro-environmental context suggests disadvantages when merely advocating for increased environmental friendly actions. Specifically, one's previous pro-environmental behaviours may provide a sense of mental licence to reduce subsequent proenvironmental behaviours or even eliminate them altogether. Therefore, we should focus on the sustainability of pro-environmental behaviours and find ways to decrease this licensing effect, to better promote environmentally friendly actions.

\section{Relationship Between the Licensing Effect and Goal Regulation}

Another important finding from the present study was that different goal foci influenced the intention to perform subsequent behaviours. More specifically, when focusing on goal progress, subsequent pro-environmental behaviours were reduced after performing a good deed. However, when focusing on goal commitment, this effect was less likely to occur, and behavioural intentions even remained environmentally friendly.

According to goal regulation theory, one behaviour can reflect divergent forms of goal pursuit (e.g., goal commitment or goal progress). Due to different goal foci, motivations can differ and lead to distinct behaviours. For instance, when individuals are focused on goal progress, they may feel a sense of achievement associated with goal fulfilment, and their cognitive resources would then switch to other unattained goals, motivating them away from the initial goal (Dhar \& Simonson, 1999; Monin \& Miller, 2001; Fishbach, \& Dhar, 2005). This can manifest as a licensing effect. However, when individuals are focused on goal commitment, past behaviour represents their commitment towards, and determination to achieve the initial goal, and their cognitive resources remain focused on this goal; thus, the licensing effect must be inhibited in order to maintain goal pursuit.

Several studies have revealed a relationship between the licensing effect and goal regulation. For instance, Merritt, Effron, and Monin (2010) observed that different goal perspectives influence the emergence, or lack thereof, of a licensing effect; subsequent behaviours will depend on that focus. A recent study revealed that when individuals consider goal progress, past moral behaviours reduced future moral actions; when focusing on goal commitment, moral behaviours helped increase subsequent moral behaviours (Susewind \& Hoelzl, 2014).

Therefore, goal regulation seems to affect the consistency of environmental behaviours. Since goal commitment reduces the licensing effect, in order to maintain pro-environmental behaviours, goal commitment focus could be a useful avenue for pro-environmental interventions. 


\section{Intervening on the Licensing Effect: Attributional Recall}

Other studies have shown that situations or clues relevant to certain goals can influence behaviours (Bargh, Chen, \& Burrows, 1996; Chartrand \& Bargh, 1996). For instance, Mukhopadhyay et al. (2008) had participants recall an experience regarding their rejection of an unhealthy food. When participants recalled reasons why they rejected the food, goal-related cognitions were reinforced, no transfer of motivation emerged, and the licensing effect was alleviated. The current study produced similar results, suggesting that recall of reasons for previous positive behaviours may be an effective licensing effect intervention. Thus, when people focus on goal progress, behaviours inconsistent with overarching goals will emerge. However, recalling attributions can help modify these tendencies by concentrating cognitive resources and maintaining motivational focus on the initial target, rather than other goals.

\section{Implications}

The current study suggested that the licensing effect could be useful for explaining the lack of consistency in people's pro-environmental behaviours. Here, we were able to broaden the research orientation regarding proenvironmental behaviours. Former studies mostly focused on predictions of pro-environmental behaviours (Tanner \& Kast, 2003), while our study was more concerned with the consistency of before-and-after environmental behaviours. Though the licensing effect did not provide the sole explanation for engagement in non-environmentally friendly behaviours, the present results suggested a need to pay close attention to factors underlying behavioural persistence. Furthermore, the licensing effect should be considered on a practical level, especially for a variety of daily life environmental behaviours. In other words, while most people are aware of the importance of being environmentally conscious, most of them are likely to be unaware of how certain phenomena, such as the licensing effect, have a negative impact on their environmental behaviours. Given the results of the present study, specifically Study 1, efforts need to be made to curtail licensing effect influences on daily behaviours. In order to help people overcome the licensing effect, the present study showed that emphasising goal commitment could be particularly effective. For example, instead of encouraging individuals to conserve water via traditional approaches (e.g., providing water saving skills, raising the price of water consumption; Seyranian, Sinatra, \& Polikoff, 2015), goal commitment interventions could be enacted. In China, residents are required to pay water consumption fees every month. Hence, one way that could work would be to let households set a monthly water consumption goal for saving water (based on their normal consumption levels). When they pay their monthly water rate, the task of goal commitment could be primed to recall their goal of saving water by using questionnaires. Thereafter, households could report on the strength of their goal persistence over time as a way to assess commitment. This might help facilitate more environmentally conscious behaviours over the longer term.

As previously stated, the licensing effect has been observed in several fields. One question that arises is whether or not licensing effects have an impact on behaviours across other domains. For instance, there is work suggesting that licensing effects can extend across prosocial behaviours and ethical decision making (Mazar \& Zhong, 2010). In one study, individuals who engaged in green product purchasing indulged in their self-interests and perpetrated a series of unethical decisions (i.e., lying to earn money). Mazar and Zhong (2010) argued that purchasing green products and subsequent honest behaviours are related to a more generalised sense of a moral self. Following this logic, we suggest that pro-environmental behaviours could give possible licence to negative behaviours in other domains. For example, a person's unwillingness to give up a seat to a needy person on a bus could result from the person's decision to take green transportation. The initial pro-environmentally friendly behaviour (taking a green bus) limited the desire to engage in a prosocial behaviour later (giving up a seat). Furthermore, proenvironmental behaviours could be construed as a form of prosocial behaviour (Nolan \& Schultz, 2015); thus, both behaviours in the aforementioned example would be indicative of general prosocial concepts. Thus, the role of goal commitment in alleviating the licensing effect could be consequential for a variety of positive behavioural outcomes.

\section{Limitations and Future Directions}

The present study sought to examine the existence of a licensing effect in a pro-environmental behavioural context, as well as tested interventions for preventing its emergence. While this was a preliminary study, some limitations should be noted. First, while college students are a useful sample (given that their current and future environmental behaviours are likely to have a great societal impact), there are issues with using one group of students from one university with regard to generalisability. Furthermore, all the participants were recruited by our posters; people saw the poster but those who chose not to participate might have fewer environmental concerns than those who did, which could influence the results. Pro-environmental interventions should be relevant to all individuals; thus, future studies should examine the licensing effect with a more diverse population of participants. Second, the ways that we measured actual pro-environmental behaviours need improvement in order to enhance their ecological validity. For example, data regarding residents' actual daily water consumption (rather than a laboratory task) would be appropriate. Finally, using questionnaire methods to assess participants' purchasing behaviours may not accurately reflect how such behaviours are performed in the real world. Thus, more authentic and convenient 
purchasing tasks (such as online shopping manipulations) could be employed in future studies. Furthermore, it would be better to select tasks of the same type for Study 3. In the current study, the experimental group performed a recall task whereas the other group performed a reading task. Perhaps the type of task performed produced the differences in outcome. Furthermore, action-oriented research should be applied in future experimental designs. That is, when conducting a study on environment protection, we could use a systematic method that includes planning (experimental design), action (taking interventions), evaluation (analysing results), and reflection (Liu \& Bernardo, 2014). For example, as well as manipulating experimental conditions to study the licensing effect, researchers could also find an enforceable way to apply what is learned in everyday life (such as using public service advertisements as the intervention of attributional recall) and measure actual outcomes for feedback on their effectiveness.

\section{Acknowledgments}

The authors gratefully acknowledge the support of the Jiangsu university philosophy social science fund project 'the mentality in the period of social transformation' (No.2015JDXM003).

\section{Supplementary Material}

To view supplementary material for this article, please visit https://doi.org/10.1017/prp.2016.6

\section{References}

Ajzen, I. (1991). The theory of planned behavior. Research in Nursing \& Health, 14, 137-144.

Bargh, J.A., Chen, M., \& Burrows, L. (1996). Automaticity of social behavior: Direct effects of trait construct and stereotype activation on action. Journal of Personality and Social Psychology, 71, 230-44.

Barr, S., Shaw, G., Coles, T., \& Prillwitz, J. (2010). 'A holiday is a holiday': Practicing sustainability, home and away. Journal of Transport Geography, 18, 474-481.

Berger, I.E. (1997). The demographics of recycling and the structure of environmental behaviour. Environment and Behaviour, 29, 515-531.

Bolton, L.E., Cohen, J.B., \& Bloom, P.N. (2006). Does marketing products as remedies create 'get out of jail free cards'? Journal of Consumer Research, 33, 71-81.

Canuel, M., Abdous, B., Bélanger, D., \& Gosselin, P. (2014). Development of composite indices to measure the adoption of pro-environmental behaviours across canadian provinces. PLoS One, 9, e101569-e101569.

Catlin, J.R., \& Wang, Y. (2012). Recycling gone bad: When the option to recycle increases resource consumption. Journal of Consumer Psychology, 23, 122-127.

Chartrand, T.L., \& Bargh, J.A. (1996). Automatic activation of impression formation and memorization goals: Nonconscious goal priming reproduces effects of explicit task in- structions. Journal of Personality \& Social Psychology, 71, 464-478.

Clot, S., Grolleau, G., \& Ibanez, L. (2014). Do good deeds make bad people? European Journal of Law and Economics, 1-23. doi:10.1007/s10657 014 9441-4

Dominicis, S.D., Fornara, F., Cancellieri, U.G., Twigger-Ross, C., \& Bonaiuto, M. (2015). We are at risk, and so what? Place attachment, environmental risk perceptions and preventive coping behaviours. Journal of Environmental Psychology, 43, 66-78.

Dhar, R., \& Simonson, I. (1999). Making complementary choices in consumption episodes: Highlighting versus balancing. Journal of Marketing Research, 36, 29-44.

Festinger, L. (1957). A theory of cognitive dissonance. A theory of cognitive dissonance. Palo Alto, CA: Stanford University Press.

Fishbach, A., \& Dhar, R. (2005). Goals as excuses or guides: The liberating effect of perceived goal progress on choice. Journal of Consumer Research, 32, 370-377.

Fishbach, A., Dhar, R., \& Zhang, Y. (2006). Subgoals as substitutes or complements: The role of goal accessibility. Journal of Personality and Social Psychology, 91, 232242 .

Fishbach, A., Zhang, Y., \& Koo, M. (2009). The dynamics of selfregulation. European Review of Social Psychology, 20, 15344.

Gans, J.S., \& Groves, V. (2009). Carbon offset provision with guilt-ridden consumers. Journal of Economics \& Management Strategy, 21, 243-269.

Hautier, Y., Tilman, D., Isbell, F., Seabloom, E.W., Borer, E.T., \& Reich, P.B. (2015). Plant ecology. Anthropogenic environmental changes affect ecosystem stability via biodiversity. Science, 348, 336-340.

Jordan, J., Mullen, E., \& Murnighan, J.K. (2009). Maintaining a less-than-perfect moral equilibrium: The paradoxical effects of past moral behaviour. Manuscript submitted for publication.

Liu, J.H., \& Bernardo, A.B.I. (2014). Social psychology for social change: Foundations for and introduction to a program of action-oriented research. Journal of Pacific Rim Psychology, $8,29-34$.

Mazar, N., \& Zhong, C.B. (2010). Do green products make us better people? Psychological Science, 21, 494-498.

Meijers, M.H.C. (2014). On justifying eco-unfriendly behaviors (Unpublished doctoral dissertation). FMG, Amsterdam School of Communication Research, Amsterdam, the Netherlands.

Merritt, A.C., Effron, D.A., \& Benoît, M. (2010). Moral selflicensing: When being good frees us to be bad. Social and Personality Psychology Compass, 4, 344-357.

Monin, B. \& Miller, D.T. (2001). Moral credentials and the expression of prejudice. Journal of Personality and Social Psychology, 81, 33-43.

Mukhopadhyay, A., Sengupta, J., \& Ramanathan, S. (2008). Recalling past temptations: An information-processing perspective on the dynamics of self-control. Journal of Consumer Research, 35, 586-599. 
Mullen, E., \& Monin, B. (2016). Consistency versus licensing effects of past moral behavior. Annual Review of Psychology, 67, 363-385.

Nolan, J.M. \& Schulz, P.W. (2015). Prosocial behavior and environmental action. In D.A. Schroeder \& W.G. Graziano, The Oxford Handbook of Prosocial Behavior (pp. 626-652). New York: Oxford University Press.

Seyranian, V., Sinatra, G.M., \& Polikoff, M.S. (2015). Comparing communication strategies for reducing residential water consumption. Journal of Environmental Psychology, 41, 8190.

Shah, J.Y., \& Kruglanski, A.W. (2003). When opportunity knocks: Bottom-up priming of goals by means and its effects on selfregulation. Journal of Personality and Social Psychology, 84, $1109-1122$.

Shah, J.Y., Kruglanski, A.W., \& Friedman, R. (2003). Goal systems theory: Integrating the cognitive and motivational aspects of self-regulation.

Shen, L. J. (2008). Character and Relationship Research on Undergraduates' Environmental Values. Attitudes and Behaviors [D]. Taiyuan: Shangxi University, 2008.

Steg, L., \& Vlek, C. (2009). Encouraging pro-environmental behaviour: An integrative review and research agenda. Journal of Environmental Psychology, 29, 309-317.
Stern, P.C. (2000). Toward a coherent theory of environmentally significant behavior. Journal of Social Issues, 56, 407-424.

Susewind, M., \& Hoelzl, E. (2014). A matter of perspective: Why past moral behavior can sometimes encourage and other times discourage future moral striving. Journal of Applied Social Psychology, 44, 201-209.

Tanner, C., \& Kast, S.W. (2003). Promoting sustainable consumption: Determinants of green purchases by Swiss consumers. Psychology \& Marketing, 20, 883-902.

Thøgersen, J. (1999). Spillover processes in the development of a sustainable consumption pattern. Journal of Environmental Psychology, 585, 3-13.

Tiefenbeck, V., Staake, T., Roth, K., \& Sachs, O. (2013). For better or for worse? Empirical evidence of moral licensing in a behavioural energy conservation campaign. Energy Policy, $57,160-171$.

Truelove, H.B., Carrico, A.R., Weber, E.U., Raimi, K.T., \& Vandenbergh, M.P. (2014). Positive and negative spillover of pro-environmental behaviour: An integrative review and theoretical framework. Global Environmental Change, 29, $127-138$.

Van der Werff, E.V.D., Steg, L., \& Keizer, K. (2014). Follow the signal: When past pro-environmental actions signal who you are. Journal of Environmental Psychology, 40, 273-282. 